ISSN 1808-3765

\title{
INFLUÊNCIA DE MANEJOS DE IRRIGAÇÃO SOBRE ASPECTOS DE ECOFISIOLOGIA E DE PRODUÇÃO DA VIDEIRA CV. SYRAH / PAULSEN 1103
}

\author{
Luís Henrique Bassoi ${ }^{1}$, Simone De Oliveira Gonçalves ${ }^{2}$, Ana Rita Leandro Dos Santos ${ }^{3}$, \\ Juliano Athayde Silva ${ }^{4}$, Araci Conceição Medrado Lima ${ }^{5}$ \\ ${ }^{1}$ Pesquisador, Embrapa Semiárido, BR 428, km 152, Caixa Postal 23, Petrolina - PE, CEP: 56302-970, e.mail: \\ lhbassoi@cpatsa.embrapa.br \\ ${ }^{2}$ Bolsista do CNPq, Mestre em Agronomia (Irrigação e Drenagem), UNESP FCA, Depto Engenharia Rural, \\ Botucatu - SP,e.mail: simone-say@hotmail.com \\ ${ }^{3}$ Professora, IF Sertão Pernambucano, Petrolina - PE, e.mail: anaritaleandro@gmail.com \\ ${ }^{4}$ Bolsista da FACEPE, Embrapa Semiárido, Petrolina - PE, e.mail: juliano.athayde@cpatsa.embrapa.br \\ ${ }^{5}$ Bolsista PIBIC CNPq, Embrapa Semiárido, Petrolina - PE, e.mail: aramedrado@hotmail.com
}

\section{RESUMO}

O manejo de irrigação pode influenciar o comportamento ecofisiológico e a produção da videira. O objetivo desse trabalho, conduzido em 2010 em Petrolina - PE, no Submédio do Vale do São Francisco, foi avaliar a influência de diferentes estratégias de manejo de irrigação no potencial de água na folha e em aspectos qualitativos e quantitativos das uvas do primeiro ciclo de produção da videira cv. Syrah/Paulsen 1103. O sistema de irrigação utilizado foi gotejamento e a lâmina de água foi estimada com base na evapotranspiração da cultura. $\mathrm{O}$ delineamento experimental utilizado foi o de blocos casualizados, em 4 repetições e com 3 tratamentos: irrigação plena, realizada durante todo o ciclo de produção; a irrigação com déficit, onde a aplicação de água foi interrompida na fase fenológica de cacho fechado; e a irrigação com déficit controlado, onde a irrigação, também interrompida na fase de cacho fechado, foi eventualmente realizada após a interrupção, de acordo com o monitoramento da água no solo. A imposição de déficit hídrico às plantas favoreceu uma maior concentração de açúcares e a redução da acidez nos frutos, contribuindo para a melhoria da qualidade das uvas para vinificação.

Palavras-chave: Vitis vinifera L., deficit hídrico, potencial de água na folha

BASSOI, L. H.; GONÇALVES, S. O.; SANTOS, A. R. L. dos; SILVA, J. A.; LIMA, A. C. M. INFLUENCE OF IRRIGATION SCHEDULINGS ON ECOPHYSIOLOGY AND YIELD ASPECTS OF GRAPEVINE CV. SYRAH / PAULSEN 1103 P

\section{ABSTRACT}

Irrigation management may influence the ecophysiological behavior and yield of grapevine. The objective of this study was to evaluate the influence of different irrigation management strategies in leaf water potential, quantitative and qualitative aspects of grapes from the first growing season of cv. Syrah / Paulsen 1103 vineyard. The experiment was carried out at experimental field of Embrapa Tropical Semiarid, in Petrolina, Pernambuco State, Brazil. Plants were irrigated by drippers and irrigation depth was estimated based on crop evapotranspiration. The experimental design was a randomized block, with 4 replications, and 
with 3 treatments: full irrigation with water application throughout the growing season; deficit irrigation with water application interrupted in the pea-sized berries phase; and regulated deficit irrigation with irrigation also interrupted in the same phenological phase but eventually performed after that according to soil water monitoring. Plant water deficit has favored a higher sugar concentration and lower acidity in the berries which are desirable for winemaking.

Keywords: Vitis vinifera L., water deficit, leaf water potential

\section{INTRODUÇÃO}

Diante da expansão da vitivinicultura, a região do Submédio do Vale do São Francisco tem evoluído no sentido de aprimorar a aplicação de práticas agronômicas visando melhorar a qualidade da produção vinícola uma vez que a composição da uva é um fator determinante para a qualidade dos vinhos dessa região.

Estratégias de manejo de irrigação com restrição hídrica vêm sendo desenvolvidas com o objetivo de manipular o crescimento vegetativo e reprodutivo da videira, uma vez que estado hídrico tem sido reconhecido como um fator determinante, que influi em todos os aspectos do crescimento e qualidade das uvas. A técnica do controle do déficit hídrico é agora utilizada de forma extensa para manipular a qualidade da uva para vinho de qualidade (Dry et al., 2001), proporcionando a maximização do uso da água pois, atualmente existe uma preocupação mundial quanto ao uso racional dos recursos hídricos, existindo uma pressão sobre os irrigantes para que haja um controle mais efetivo da irrigação (Loveys et al., 2004). Vinhedos submetidos à irrigação plena durante todo o ciclo de produção são desfavoráveis a produção de vinhos de qualidade. A imposição de um estresse hídrico moderado à videira em determinadas fases fenológicas tem um impacto positivo sobre a concentração de açúcares, acidez, pH e compostos fenólicos no mosto (Deloire et al., 2004), que são os responsáveis pela cor, aroma, corpo e estrutura dos vinhos tintos.

Assim, o objetivo desse trabalho foi o de avaliar a influência de diferentes estratégias de manejo de irrigação no comportamento ecofisiológico e na produção de uvas pela videira Syrah / Paulsen 1103, em Petrolina - PE.

\section{MATERIAL E MÉTODOS}

O experimento foi instalado no Campo Experimental de Bebedouro (latitude $9^{\circ} 8^{\prime}$ $8,9^{\prime \prime} \mathrm{S}$, longitude $40^{\circ} 18^{\prime} 33,6^{\prime \prime} \mathrm{O}$, altitude $373 \mathrm{~m}$ ), pertencente a Embrapa Semiárido, em Petrolina-PE. A videira (Vitis vinifera L.) cultivar Syrah foi enxertada sobre o porta-enxerto Paulsen 1103, sendo as mudas do porta-enxerto obtidas por meio de estaquia. O plantio foi feito em 30 de abril de 2009 no espaçamento de $1 \mathrm{~m}$ entre plantas e $3 \mathrm{~m}$ entre fileiras e a condução das plantas foi feita no sistema de espaldeira, com duplo cordão esporonado. $\mathrm{O}$ período de formação do parreiral (crescimento vegetativo) ocorreu até 13 de abril de 2010, quando ocorreu a poda de produção do primeiro ciclo. $\mathrm{O}$ solo da área em questão é classificado como Argissolo Vermelho Amarelo Eutrófico Latossólico, textura média (Silva, 2005). O sistema de irrigação utilizado foi o de gotejamento, com emissores espaçados a 0,5 $\mathrm{m}$ na linha de plantio e com vazão de $2,5 \mathrm{~L} \mathrm{~h}^{-1}$, a uma pressão de serviço de $100 \mathrm{kPa}$, e aferida em teste de vazão no campo. O cálculo da irrigação foi com base na evapotranspiração de 
referência (ETo), estimada pelo método de Penman-Monteith FAO, por meio de parâmetros medidos pela estação agrometeorológica automática instalada a cerca de $50 \mathrm{~m}$ da área do experimento. Para a determinação dos valores de evapotranspiração da cultura (ETc),foram adotados os valores de Kc recomendados por Silva (2005) e Bassoi et al. (2007). Cada parcela foi composta por 48 plantas, sendo 2 fileiras com 24 plantas.

O delineamento experimental adotado foi de blocos casualizados, em 4 repetições, com 3 tratamentos: 1- irrigação plena (IP), onde a irrigação foi feita para repor a lâmina correspondente à quantidade de água evapotranspirada, sem a restrição de água às videiras durante todo o ciclo de produção; 2 - irrigação deficitária (ID), onde a aplicação de água foi interrompida desde 45 dias após a poda de produção - dapp (fase fenológica de cacho fechado) até a colheita (6 de agosto de 2010, 115 dapp); 3 - irrigação com déficit controlado (IDC), onde a aplicação de água foi interrompida desde fase fenológica de cacho fechado (45 dapp), mas realizada ( 23 e 23 de junho, 3, 7 e 12 de julho e 2 de agosto, respectivamente aos $70,71,87,90,111$ dapp), para o aumento da umidade do solo $\left(\theta, \mathrm{m}^{3} \mathrm{~m}^{-3}\right)$ na profundidade efetiva do sistema radicular $(0,6 \mathrm{~cm})$, de acordo com Silva (2005), conforme as condições de umidade do solo monitorada semanalmente pela técnica de moderação de nêutrons, nas profundidades de $0,15,0,30,0,45,0,60,0,75,0,90,1,05$ e $1,20 \mathrm{~m}$.

O potencial hídrico foliar de base $\left(\Psi_{\mathrm{pd}}, \mathrm{MPa}\right)$ foi medido em 8 e 22 de junho de 2010 (56 e 70 dapp) no momento em que a fase fenológica identificada foi a de cacho fechado; em 8 e 21 de julho de 2010 (86 e 99 dapp) na fase de maturação; e em 4 de agosto de 2010 (113 dapp), quando os frutos já estavam no ponto de colheita, sempre entre $3 \mathrm{~h} 00 \mathrm{~min}$ a.m. e 4h 30 min a.m.. Nessas ocasiões, foram coletadas amostras de folhas adultas, da porção mediana de ramos produtivos ( 3 folhas por planta), sendo armazenadas em saco plástico para reduzir a perda de água do material vegetal para o meio ambiente, e utilizadas imediatamente após a retirada para a medição do $\Psi_{\text {pd }}$ por meio da câmara de pressão de Scholander.

Na colheita, realizada em 6 de agosto de 2010 (115 dapp), foi determinado o número e a massa total de cachos por planta. A partir desses dados foram estimados o rendimento médio total da produção $\left(\mathrm{Kg} \mathrm{ha}^{-1}\right)$ e a massa individual de cachos. Posteriormente, foram separadas 100 bagas do engaço, mantendo-se o pedicelo, pesadas em balança analítica digital e colocadas em uma proveta graduada de $500 \mathrm{ml}$. As provetas foram aferidas com $300 \mathrm{ml}$ de água. A variação do volume de água foi considerada como sendo o volume de 100 bagas. Após maceradas foi possível a obtenção do volume do mosto das mesmas 100 bagas por meio de proveta graduada. Ainda foram realizadas no mosto, análises de teor de sólidos solúveis totais $\left({ }^{\circ}\right.$ Brix), utilizando-se de um refratômetro portátil marca ATAGO modelo Pocket PAL1, acidez total titulável - expressa em $\mathrm{g} \mathrm{L}^{-1}$ de ácido tartárico, conforme metodologia descrita por Pregnolatto (1985), e a determinação do $\mathrm{pH}$ por meio de pHmetro digital marca TECNAL.

\section{RESULTADOS E DISCUSSÃO}

\subsection{Precipitação pluvial}

Durante o ciclo de produção da videira foram registrados $45,7 \mathrm{~mm}$ de precipitação. No início da fase de maturação (76 dapp), até o momento da colheita (115 dapp), a ocorrência de precipitação registrada foi de 13,2 mm, com o último dia de chuva aos 107 dapp, sete dias antes da colheita. 
A evapotranspiração da cultura (ETc) acumulada durante o ciclo de produção foi de $241,1 \mathrm{~mm}$, com valor máximo diário de 4,5 $\mathrm{mm}$ ocorrido aos 80 dapp, e valor médio de 2,6 $\mathrm{mm} \mathrm{dia}{ }^{-1}$. A lâmina bruta total aplicada durante o ciclo de produção foi de $312,1 \mathrm{~mm}$. Bassoi et al (2007) encontraram valores de ETc diária de 2,5 $\mathrm{mm} \mathrm{dia}^{-1}$ (próximo à colheita) a 4,2 $\mathrm{mm}$ $\mathrm{dia}^{-1}$ (fase de pegamento do fruto) em videira Syrah / 1103 P cultivada em espaldeira, e na mesma área experimental.

\subsection{Umidade do solo}

Até a profundidade de $0,6 \mathrm{~m}, \theta$ apresentou uma variação bastante distinta entre os tratamentos de manejo de irrigação (Figura 1). A partir da interrupção da aplicação de água (45 dapp), $\theta$ decresceu gradativamente nos tratamentos IDC e ID, sendo que após a aplicação de água no tratamento IDC (70, 71, 87, 90, 111 dapp), observou-se a elevação de $\theta$ até $0,30 \mathrm{~m}$ de profundidade. Silva (2005), na mesma área experimental, determinou que a videira cv. Syrah / $1103 \mathrm{P}$ apresentou a profundidade efetiva do sistema radicular de 0,6 m. Assim, essa camada de 0,6 m contribuiu de maneira considerável para os processos de evapotranspiração e drenagem. Nas camadas mais profundas do solo $(0,75,0,90,1,05$ e $1,20 \mathrm{~m})$, a variação de $\theta$ foi praticamente a mesma em todos os tratamentos.
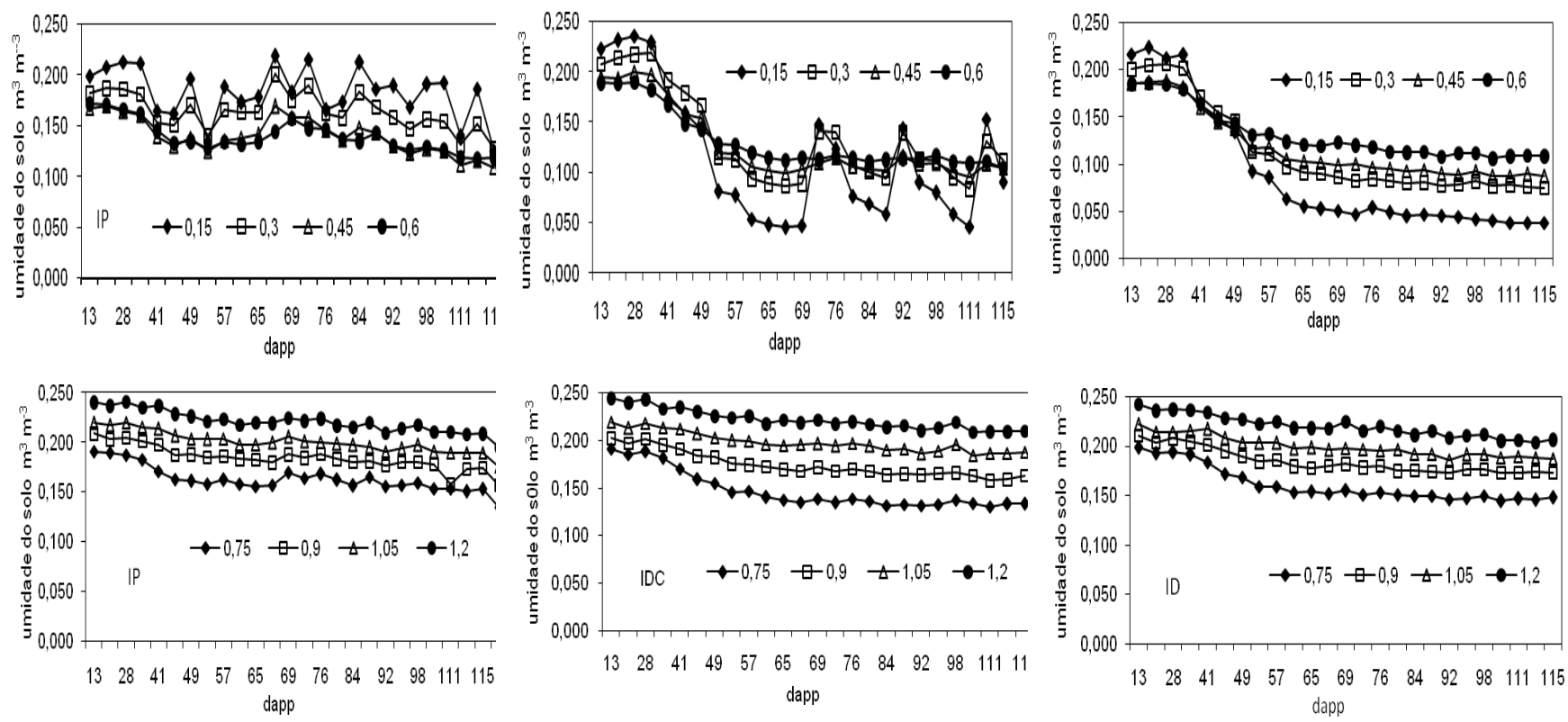

Figura 1. Umidade do solo $(\theta)$ à $0,15,0,30,0,45,0,60,0,75,0,90,1,05$ e $1,20 \mathrm{~m}$ de profundidade, em função dos tratamentos irrigação plena (IP), irrigação com déficit controlado (IDC) e irrigação com déficit (ID).

\subsection{Potencial hídrico foliar de base}

Aos 56 dapp, 11 dias após a interrupção da irrigação nos tratamentos ID e IDC, não houve diferenças entre os valores de $\Psi_{\mathrm{pd}}$ (Figura 2). Aos 70 dapp, foi registrado um decréscimo nos valores de $\Psi_{\mathrm{pd}}$ nos três tratamentos, que pode ser justificado pela ocorrência, no dia anterior, de $8,13 \mathrm{~mm}$ de precipitação. Nesse dia, o $\Psi_{\text {pd }}$ no tratamento IP foi menor que os dos demais tratamentos, indicando um maior teor de água nas folhas das plantas sob irrigação plena. Aos 70 e 71 dapp, foram realizadas irrigações no tratamento IDC. No entanto, 
comportamento semelhante aos 70 dapp foi observado aos 86 dapp. Aos 99 dapp, 54 dias após a interrupção da irrigação, foi registrado o maior valor médio de $\Psi_{\mathrm{pd}}$ no tratamento ID, mas sem diferir de IDC e IP. Aos 87 e 90 dapp foram realizadas irrigações no tratamento IDC o que pode ter contribuído para a não diferença entre tratamentos. Aos 113 dapp, as plantas com restrição hídrica apresentavam menor teor de água na folhas que as plantas sob irrigação plena. Aos 111 dapp, houve irrigação nas plantas do IDC.

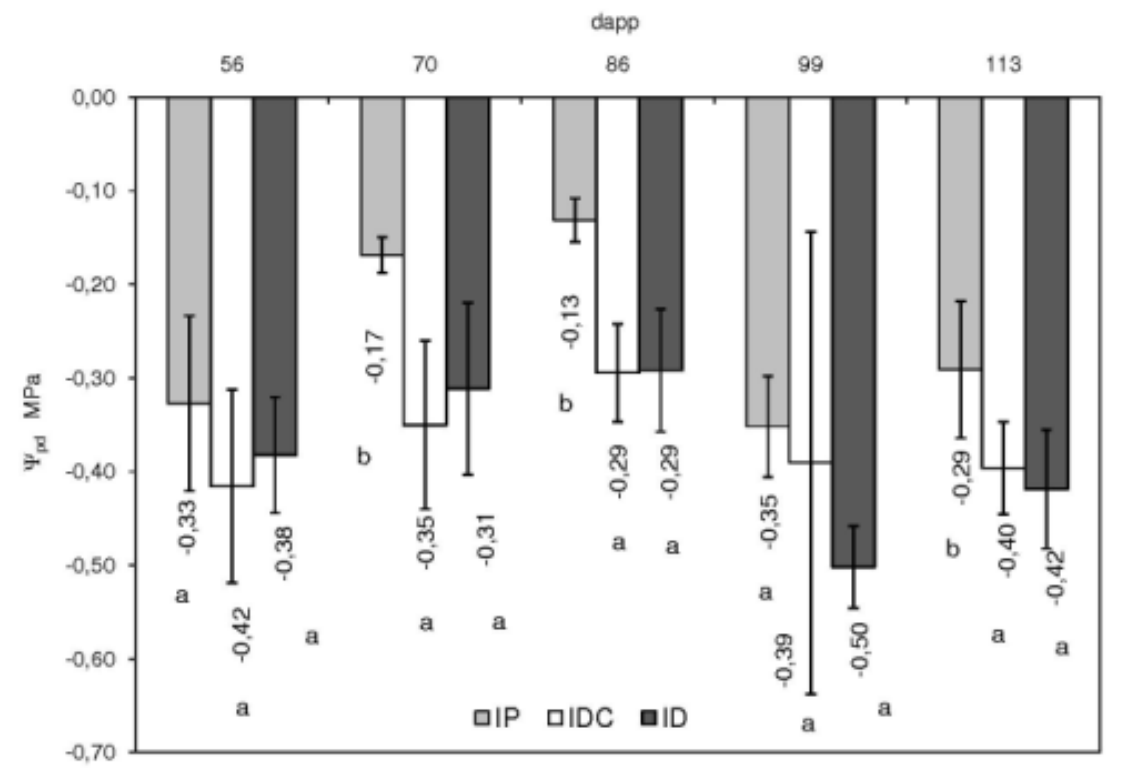

Figura 2. Potencial hídrico foliar de base $\left(\Psi_{\mathrm{pd}}\right)$ da videira Syrah/Paulsen 1103, nos tratamentos irrigação plena (IP), irrigação com déficit controlado (IDC) e irrigação deficitária (ID), aos 56, 70, 86, 99 e 113 dias após a poda de produção (dapp).

\subsection{Aspectos quantitativos e qualitativos da produção}

Em relação ao número total de cachos por parcela, número total de cachos por planta, massa total de cachos por planta e rendimento total de cachos, os tratamentos de irrigação não apresentaram, estatisticamente, diferença significativa entre si (Tabela 1). Os tratamentos IP e IDC apresentaram maior massa individual de cachos por planta e volume de 100 bagas, enquanto que somente o tratamento IP apresentou maior massa de 100 bagas.

O tamanho final da baga determina a qualidade da baga, por meio da relação entre área da película e volume do mosto. O déficit hídrico entre a antese (abertura das flores) e o veraison (início do amadurecimento das bagas) diminui o tamanho final da baga de modo irreversível, mesmo que haja um umedecimento após o veraison. Entre o veraison e a colheita, as bagas aumentam em volume, peso e conteúdo de açúcares. A interrupção da aplicação de água nesse trabalho ocorreu aos 45 dapp, na fase de cacho fechado (antes do veraison, ocorrido aos 85 dapp), e a disponibilidade de água no solo nos tratamentos IDC e ID (Figura 1) foi reduzida principalmente até a profundidade de solo de $0,6 \mathrm{~m}$. Esse déficit hídrico causou uma redução do conteúdo de água na folha das plantas dos mesmos tratamentos (Figura 2).

Consequentemente, os valores de massa individual dos cachos, massa de 100 bagas e volume do mosto de 100 bagas foram menores no tratamento ID, seguidos pelo IDC e IP (Tabela 1). 
Tabela 1. Aspectos quantitativos e qualitativos da produção da videira cultivar Syrah/1103P, em função dos tratamentos irrigação plena (IP), irrigação com déficit controlado (IDC) e irrigação deficitária (ID). Petrolina, 2010.

\begin{tabular}{|c|c|c|c|}
\hline Variáveis & IP & IDC & ID \\
\hline $\mathrm{N}^{\mathrm{o}}$ total de cachos por parcela (un) & $146,25 \mathrm{a}$ & $114,75 \mathrm{a}$ & $143,25 \mathrm{a}$ \\
\hline $\mathrm{N}^{\mathrm{o}}$ total de cachos por planta (un) & $12,36 \mathrm{a}$ & $10,27 \mathrm{a}$ & $12,76 \mathrm{a}$ \\
\hline Massa individual de cachos por planta $(\mathrm{g})^{1}$ & $177 \mathrm{a}$ & $165 a, b$ & $150 \mathrm{~b}$ \\
\hline Massa total de cachos por planta $(\mathrm{kg})$ & $2,16 \mathrm{a}$ & $1,70 \mathrm{a}$ & $1,91 \mathrm{a}$ \\
\hline Rendimento total da produção $\left(\mathrm{Kg} \mathrm{ha}^{-1}\right)$ & $7.212 \mathrm{a}$ & $5.677 \mathrm{a}$ & $6.367 \mathrm{a}$ \\
\hline Massa de 100 bagas $(\mathrm{g})^{1}$ & $175 \mathrm{a}$ & $150 \mathrm{~b}$ & $147 \mathrm{~b}$ \\
\hline Volume do mosto de 100 bagas $(\mathrm{mL})^{2}$ & $62,91 \mathrm{a}$ & $56,66 \mathrm{ab}$ & $52,91 \mathrm{~b}$ \\
\hline Acidez total titulável $\left(\mathrm{g} \mathrm{L}^{-1}\right)^{1}$ & $9,78 \mathrm{a}, \mathrm{b}$ & $10,05 \mathrm{a}$ & $9,26 \mathrm{~b}$ \\
\hline $\mathrm{pH}$ & $3,17 \mathrm{a}$ & $3,17 \mathrm{a}$ & $3,2 \mathrm{a}$ \\
\hline Teor de sólidos solúveis $\left({ }^{\circ}\right.$ Brix $)$ & $19,55 \mathrm{a}$ & $20,20 \mathrm{a}$ & $20,00 \mathrm{a}$ \\
\hline
\end{tabular}

${ }^{1,2}$ Médias na mesma linha seguidas pela mesma letra não diferem entre si a, respectivamente, $5 \%$ e $10 \%$ de probabilidade pelo teste Tukey.

Os valores encontrados para o rendimento total da produção $\left(\mathrm{kg} \mathrm{ha}^{-1}\right)$ foram inferiores aos encontrados por Favero et al. (2008), em ciclos de outono-inverno (fevereiro a julho) em 2005 (6158 $\left.\mathrm{kg} \mathrm{ha}^{-1}\right)$ e $2006\left(7704 \mathrm{~kg} \mathrm{ha}^{-1}\right)$ em Minas Gerais, mas superiores à produção reportada por Bassoi et al. (2007) na mesma área experimental em 2004 (4774 kg $\left.\mathrm{ha}^{-1}\right)$.

Quanto aos aspectos físico-químicos, as uvas do tratamento ID apresentaram menor acidez total titulável, mas não correram diferenças quanto $\mathrm{pH}$ e teor de sólidos solúveis totais (Tabela 1).

A imposição de um déficit hídrico moderado à videira em determinadas fases fenológicas tem um impacto positivo sobre a concentração de açúcares, acidez, $\mathrm{pH}$ e compostos fenólicos no mosto (Deloire et al., 2004), que são os responsáveis pela cor, aroma, corpo e estrutura dos vinhos tintos. O déficit hídrico geralmente resulta em bagas menores e modifica a composição do fruto. ROBY et al. (2004) avaliaram o efeito do tamanho das bagas e déficit hídrico na composição de uvas Cabernet Sauvignon e observaram que o acúmulo de sólidos solúveis depende do tamanho das bagas e é favorecido por estresse hídrico moderado (entre -1,2 e -1,4 MPa). Em condições de estresse hídrico, o aumento no teor de sólidos solúveis é decorrente da concentração de açúcares como consequência do menor tamanho da baga. Neste caso, as bagas são menores devido à menor disponibilidade de água para a elongação celular (Esteban et al., 2002). Conforme Amorim et al. (2005), a superioridade do valor de $\mathrm{pH}$ pode ocorrer pela maior absorção de minerais (cátions) favorecida pela maior quantidade de água aplicada. No caso da uva para vinho o valor de $\mathrm{pH}$ recomendável para o mosto é no máximo 3,30. Os valores médios encontrados nesse trabalho são considerados satisfatórios, pois segundo Ribéreau-Gayon et al. (2004), para a produção de vinho de qualidade, é necessário que a uva atinja no mínimo $18^{\circ}$ Brix de sólidos solúveis totais. Esses mesmos valores também foram superiores ao valor médio de $16,5^{\circ}$ Brix, observado durante quatro safras de verão por Souza et al. (2002). Amorim et al. (2005) relataram um valor de 21,75 ${ }^{\circ}$ Brix 15 dias antes da colheita em experimento realizado em Minas Gerais para avaliar a produção extemporânea da cultivar Syrah. 


\section{CONCLUSÕES}

A prática do manejo de irrigação com déficit hídrico, a partir da fase fenológica de cacho fechado, alterou o comportamento ecofisiológico da videira cv. Syrah / 1103 P em seu primeiro ciclo de produção em Petrolina - PE, e reduziu a massa individual de cachos, a massa e o volume de 100 bagas, e a acidez total titulável do mosto. O teor de sólidos solúveis totais e o pH do mosto não foram influenciados pelos tratamentos com déficit hídrico e déficit hídrico controlado.

\section{REFERÊNCIAS}

AMORIM, D. A. de; FAVERO, A. C.; REGINA, M. de A. Produção extemporânea da videira, cv. Syrah, nas condições do Sul de Minas Gerais. Revista Brasileira de Fruticultura, Jaboticabal, v. 27, n. 2, p. 327-331, ago.2005.

BASSOI, L. H. ; et. al. Preliminary results of a long term experiment about RDI and PRD irrigation strategies in wine grape in São Francisco Valley, Brazil. Acta Horticulturae, Leuven, n.. 754, p. 275-282, 2007.

DELOIRE, A.; et. al. Vine and water a short review. Journal International des Sciences de la Vigne et du Vin, Bordeaux, v. 38, n. 1, p. 1-13, 2004.

DRY, P. R.; et. al. Strategic irrigation management in Australian vineyards. Journal International des Sciences de la Vigne et du Vin, Bordeaux, v. 35, n. 3, p.129-139, 2001.

ESTEBAN, M.A.; VILLANUEVA, M.J.; LISSARRAGUE, J.R. Relationships between different berry components in Tempranillo (Vitis vinifera L.) grapes from irrigated and nonirrigated vines during ripening. Journal of the Science of Food and Agriculture, Chichester, v.82, p.1136-1146, 2002.

FAVERO, A. A.; et. al. Viabilidade de produção da videira 'Syrah', em ciclo de outono inverno, na região sul de Minas Gerais. Rev. Bras. Frutic., Jaboticabal, v. 30, n. 3, p. 685$690,2008$.

GU, S.; et. al. Effects of irrigation amount on water relations, vegetative growth, yield and fruit composition of Sauvignon blanc grapevines under partial rootzone drying and conventional irrigation in the San Joaquin Valley of California, USA. Journal of Horticultural Science and Biotechnology, v.79, p.26-33, 2004.

LOVEYS, B. R.; STOLL, M.; DAVIES, W. J. Physiological approaches to enhance water use efficiency in agriculture: exploiting plant signalling in novel irrigation practice. In: BACON, M. A. (Ed.) Water use efficiency in plant biology. Boca Raton: CRC Press , 2004. p.113-138.

OJEDA, H.; DELOIRE, A.; CARBONNEAU, A. Influence of water deficits on grape berry growth. Vitis, Siebeldingen, v. 40, n. 3, p. 141-145, 2001. 
PREGNOLATTO, W.; PREGNOLATTO, N.P. (Coord.). Normas analiticas do Instituto Adolfo Lutz: metodos quimicos e fisicos para analise de alimentos. 3.ed. São Paulo: Instituto Adolfo Lutz, 1985. v.1.

RIBÉRAU-GAYON, J.; et. al. Traité d'enologie: 1.Microbiologie Du vin - vinifications. 5. ed. Paris: Dunod. 2004. 661p.

ROBY, G.; et. al. Berry size and vine water deficits as factors in winegrape composition: anthocyanins and tannins. Australian Journal of Grape and Wine Research, Adelaide, v.10, p.100-107, 2004.

SILVA, J. A. M. Irrigação lateralmente alternada e com deficit hídrico na videira cv. Petite Syrah. 2005. 99 f. Dissertação (Mestrado), Universidade Federal de Viçosa. Viçosa, MG. 